\title{
Electroconvulsive Therapy for Trauma-Related Nightmares: A Case Report and Commentary
}

\author{
Oliver Shore $^{1}$, Pauline Chen ${ }^{1}$, Tessy Korah ${ }^{1}$ \\ 1. Psychiatry, University of Florida, Gainesville, USA
}

Corresponding author: Tessy Korah, korah@ufl.edu

\begin{abstract}
Trauma-related nightmares (TRN), one of the most reported symptoms of posttraumatic stress disorder (PTSD), may not always respond to current pharmacologic and therapeutic treatments. Validity of electroconvulsive therapy (ECT), which is used worldwide in clinical treatment for a broad range of neuropsychiatric conditions, is investigated as a potential therapeutic option for TRN in this report. A case of a 39-year-old male with a history of severe combat-related PTSD, major depressive disorder, history of traumatic brain injury, suicidal ideations, and persistent TRN is discussed here. Successful treatment outcome of this case with six sessions of right unilateral ECT is presented. On initial presentation, the patient had a Patient Health Questionnaire-9 (PHQ-9) score of 27 and a Posttraumatic Stress Disorder Checklist for DSM-5 (PCL-5) score of 77. After six sessions of ECT, the patient had a PHQ-9 score of 3 and a PCL-5 score of 45 . Furthermore, the rationale and potential mechanisms of action underlying the ECT treatment for treatment-resistant PTSD and TRN are also reviewed in this report.
\end{abstract}

Categories: Psychiatry

Keywords: trauma-related nightmares, post-traumatic stress disorder, electroconvulsive therapy, traumatic brain injury, trauma-focused psychological therapy

\section{Introduction}

Trauma is an emotional response to a shocking or overwhelming event that threatens one's physical, mental, or emotion safety. The event can be experienced first-hand, witnessed in person, happen to a close relation, or occur following repeated exposure to disturbing details of traumatic events [1]. A majority of those exposed to a traumatic event will have a psychological reaction that can manifest into four broad symptoms groups: intrusion, avoidance, hyperarousal, and negative cognition and mood. Intrusive symptoms consist of recurrent involuntary trauma-related memories, flashbacks, and nightmares that cause physiologic and psychologic distress. Avoidant symptoms are behavioral changes that are enacted to avoid internal and external trauma reminders. Hyperarousal symptoms consist of irritable and angry outbursts without appropriate provocation, reckless behavior, hypervigilance, exaggerated startle, and sleep disturbance. Negative cognition and mood symptoms can include amnesia for the traumatic event, negative emotional states, negative beliefs about oneself, others, or the world, anhedonia, and a loss of positive emotions [1]. Additionally, approximately half of people with posttraumatic stress disorder (PTSD) also have

Review began 06/10/2021 Review ended 07/11/2021 Published 07/21/2021

\section{๑) Copyright 2021}

Shore et al. This is an open access article distributed under the terms of the Creative Commons Attribution License CC-BY 4.0., which permits unrestricted use, distribution, and reproduction in any medium, provided the original author and source are credited.

a diagnosis of major depressive disorder (MDD) [2].

\section{Trauma-related nightmares}

Trauma-related nightmares (TRN), one of the most reported symptoms of PTSD, are repeated, dysphoric dreams that cause significant distress and functional impairment [3]. Nightmares occur in association with multiple psychiatric diseases including acute stress disorder, MDD, bipolar disorder, and psychotic disorders and can be significant causes of distress [1,4]. TRN can also coincide with other sleep disturbances including insomnia due to a fear of falling asleep [5]. TRN are commonly experienced at similar rates throughout populations. Combat veterans with PTSD (52.4\%) and survivors of the Oklahoma City Bombing (50\%) experienced nightmares at similar rates $[5,6]$. Moreover, Krakow et al. found that $60 \%$ of PTSD patients reported the frequency of nightmares as many as six nights per week [7].

Physiologic mechanisms associated with nightmares and PTSD are potentially associated with limbic, paralimbic, and prefrontal dysfunction [8]. Limbic system dysfunction may lead to overactive arousal, and prefrontal dysfunction hinders appropriate de-escalation. The hippocampus and amygdala detect, generate, and maintain fearful emotions. The hippocampus maintains memories of conditioned fear, and the amygdala encodes, retrieves, and elicits a physiologic fear response [8,9]. The hippocampus and amygdala also contribute to dream production and dream content and consequently, the hippocampal dysfunction has been reported to cause short, stereotyped, and repetitive dreams. Recurrent trauma-related nightmares may be due to a dysfunctional hippocampus causing repetitive traumatic dreams and an amygdala that is sensitive and responsive to the fear-inducing elements portrayed in the dream [9].

\section{Treatment strategies for TRN}


Although treatment guidelines for PTSD vary according to each psychiatric organization, there is a general consensus that trauma-focused psychological therapy (TFPT) is effective and should be utilized as a firstline therapy. TFPT encompasses multiple therapies including exposure therapy, cognitive processing therapy, trauma-focused cognitive behavioral therapy, and eye movement desensitization and reprocessing [10].

Behavioral therapies have been found to be effective in treating TRN. Image rehearsal therapy, where participants practice envisioning pleasant imagery and use these images to modify nightmares, significantly reduced the frequency of nightmares in sexual assault survivors by $60 \%$. However, it did not result in complete TRN remission, with patients still experiencing nightmares 1.3 nights per week [11]. Exposure, relaxation, and rescripting treatment (ERRT) is a similar behavioral therapy that focuses on modifying physiological, behavioral, and cognitive channels of anxiety before bedtime, which has been shown to be helpful in reducing TRN in several case series [12]. Selective serotonin reuptake inhibitors (SSRIs) and selective serotonin-norepinephrine reuptake inhibitors (SNRIs) have the greatest evidence supporting their use in PTSD treatment, and prazosin can be used with SSRIs or SNRIs to reduce nightmares and sleep disturbances [3]. Prazosin has been shown to decrease recurrent and distressing TRN by $50 \%$ in combat veterans with PTSD although nightmares were still experienced 2.3 nights per week [13]. Prazosin has alpha1 adrenergic-blocking properties that antagonizes norepinephrine outflow from the amygdala and may otherwise contribute to autonomic stimulation, disrupted sleep, and corticotropin-releasing factor release [3]. Pharmacotherapy is typically used for TRN in conjunction with TFPT or when TFPT is unavailable [10]. Pharmacotherapy is generally effective at decreasing hyperarousal and mood symptoms, but is less effective at reducing intrusive and avoidant symptoms [3].

Existing pharmacologic and behavior therapies for TRN, while effective in reducing TRN frequency, rarely allow patients to achieve full remission. Patients continue to experience nightmares multiple nights a week that disrupt healthy sleep and negatively affect quality of life. Full remission of TRN remains a challenging but an important treatment goal.

\section{Rationale for electroconvulsive therapy treatment for TRN}

Electroconvulsive therapy (ECT) is a safe and effective treatment modality for a variety of psychiatric conditions including depression, mania, schizophrenia/ schizoaffective disorder, and catatonia. Diagnostic indications for ECT usage vary geographically, with schizophrenia and schizoaffective disorders being the number one indication worldwide [14].

In the United States, affective disorders are the main diagnostic indication for ECT, accounting for $72 \%-92 \%$ of treatments while schizophrenia and/or schizoaffective disorders account for the remainder of ECT treatments, roughly $8 \%-29 \%$. Non-mood and psychotic disorders account for less than $1 \%$ of ECT treatments [14].

Patients with major depressive episodes achieve a $60 \%$ remission rate and a $70 \%$ response rate with ECT. Mania that is not responsive to pharmacologic therapy has been effectively treated with ECT and is preferred in severe mania variants like catatonic excitement [15]. Although antipsychotic medications outperform ECT in treating schizophrenia and other psychotic disorders, the combination of ECT and antipsychotic therapy appears to reduce symptom duration and improve remission rates in medicationresistant patients $[3,15]$. Unlike with depression and psychotic disorders, limited research has been conducted evaluating ECT for PTSD.

\section{Case Presentation}

A 39-year-old male with an approximate 13-year history of PTSD, MDD, alcohol use disorder in remission, obstructive sleep apnea (OSA), and a history of traumatic brain injury (TBI) presented for worsening depression, severe combat-related PTSD, and suicidal ideations (SI). The patient had been tried on several medications prior to admission including prazosin, doxepin, amitriptyline, bupropion, duloxetine, fluoxetine, mirtazapine, paroxetine, propranolol, quetiapine, risperidone, and topiramate without relief from his symptoms. Patient trialed prazosin for a minimum of four months and up to $25 \mathrm{mg}$ without benefit. He was also engaged in group and individual therapies that included present-centered therapy (PCT), prolonged exposure (PE), trauma-focused, and acceptance and commitment therapy (ACT) for at least two years.

On initial presentation, he reported SI daily and woke up screaming from nightmares every night, which severely impacted his quality of life. On Patient Health Questionnaire-9 (PHQ-9), he scored 27 and his Posttraumatic Stress Disorder Checklist for DSM-5 (PCL-5) score was 77, indicating severe symptoms of MDD and PTSD, respectively. His medication regimen while inpatient was adjusted to include venlafaxine $225 \mathrm{mg}$ daily, aripiprazole $5 \mathrm{mg}$ daily, trazodone $200 \mathrm{mg}$ nightly, mirtazapine $15 \mathrm{mg}$ nightly, and cyproheptadine $8 \mathrm{mg}$ nightly. Cyproheptadine was later switched to clonidine $0.1 \mathrm{mg}$ with minimal benefit. The patient was also seen one to two times a week for individual cognitive behavioral therapy (CBT) and ACT. He additionally regularly attended group therapy sessions while inpatient. However, his depressive and PTSD symptoms did not improve significantly. He continued to report SI daily and woke up to vivid TRN. 
The night staff would corroborate the nighttime awakenings with reports of hearing the patient scream "abort, abort!"

Due to the severity of his depressive symptoms and despite significant improvement with therapeutic and medication management, the patient was treated with right unilateral ECT for MDD. He underwent ECT three times a week on Monday, Wednesday, and Friday. After his third ECT session, the patient reported diminished SI and no longer reported TRN. Night staff no longer reported nighttime awakenings or screaming. Furthermore, the patient reported improved quality of sleep after ECT session 3 as he was no longer waking up at night due to TRN.

ECT was greatly beneficial for the patient's depression as evidenced by the patient's report of improving SI and a PHQ-9 score of 3 on the day of discharge. By ECT session 6, the patient no longer reported SI and continued to deny reoccurrence of TRN. The patient was discharged after ECT session 6 with a plan to continue outpatient ECT sessions. On the day of discharge, his PCL-5 score was 45 , which was still suggestive of PTSD symptoms, but lower than the admission score of 77 (Table 1).

\begin{tabular}{|l|l|l|}
\hline & PHQ-9 & PCL-5 \\
\hline Admission & 27 & 77 \\
\hline Discharge & 3 & 45 \\
\hline
\end{tabular}

\section{TABLE 1: Patient's admission and discharge PHQ-9 and PCL-5 scores}

PHQ-9, Patient Health Questionnaire-9; PCL-5, Posttraumatic Stress Disorder Checklist for DSM-5

\section{Discussion}

Despite ECT's long-time use, the mechanism of action remains largely unclarified. Theories proposed to explain the physiological basis of ECT include changes to the (a) brain morphology, (b) brain activity, (c) neurotransmitter signaling, and (d) hypothalamic-pituitary-adrenal (HPA) normalization [16-18]. Tendolkar et al. found that bitemporal ECT increased hippocampal and amygdala volume bilaterally, but could not correlate these changes with the clinical response to treatment [16]. Dukart et al. found that right unilateral ECT increased gray matter volume (GMV) throughout in the right hemisphere, and particularly in the subgenual cortex, hippocampus, amygdala, and anterior temporal pole while decreased GMV in the right prefrontal cortex [19]. Furthermore, studies have correlated improved depression scores with deactivation of the bilateral frontal and anterior temporal cortices, deactivation of the right prefrontal cortex and left posterior frontal cortex, or deactivation in the frontopolar gyrus, superior temporal gyrus, and amygdala following ECT [17]. Other studies have shown that ECT-induced seizures acutely activate the HPA axis while decreasing cortisol response to dexamethasone following ECT treatment, suggesting that ECT may normalize function by allowing for HPA suppression by the forebrain $[17,18]$.

Considering that ECT is a safe procedure and is associated with decreased all-cause mortality in patients, it is not only a treatment modality for depression, but may also be a reasonable treatment modality for TRN, particularly in patients who do not respond to behavioral and pharmacologic therapies $[14,15]$.

As suggested by the PCL-5 scoring, the PTSD symptoms of the patient in the current case lessened markedly after undergoing six ECT sessions. This case does not suggest complete resolution of PTSD symptoms, but does suggest benefit for TRN as the patient no longer had nighttime awakenings or reports of TRN with continued sessions of ECT. As evidenced in this case, when pharmacological and therapeutic managements have been exhausted, ECT may be a procedural option to consider for TRN. However, this therapeutic option is scarcely explored. There have only been a handful of clinical studies and case reports suggesting beneficial effects of ECT on PTSD. In a literature search by Youssef et al., in 2017, only three retrospective studies, one prospective uncontrolled clinical trial, and five case reports were found [8].

There is also consideration that there may have been improvement in TRN secondary to improvement in the patient's depressive symptoms as nightmares are not limited to PTSD [4]. However, the patient reported cessation of TRN by ECT session 3 prior to the resolution of SI. This suggests that ECT may have a direct impact on TRN rather than an indirect impact by improving depressive symptoms.

The impact of right unilateral versus bitemporal ECT for TRN and/or PTSD is unclear. However, per previous case studies and this case report, both types have been associated with the reduction of PTSD symptoms. One retrospective case control study suggested that ECT was associated with reduction in suicidality, cardiovascular, and all-cause mortality in individuals with MDD and PTSD [20]. 
Like with depression, the mechanism for how ECT may reduce TRN and/or PTSD symptoms is still unclear. However, one hypothesis suggests that ECT impairs reconsolidation of reactivated, emotionally aversive memories and therefore reduces PTSD symptoms [21]. Given that TRN may be due to a dysfunctional hippocampus and overactive amygdala, the reported changes caused by ECT treatment may in fact be efficacious in treating TRN $[8,9,16,18]$. Further research studies are needed to determine the efficacy of ECT in TRN and PTSD, as well as research in identifying the mechanisms involved.

\section{Conclusions}

Although ECT has primarily been utilized for depression, mania, and psychotic disorders, there may be benefit in utilizing ECT for TRN. Nevertheless, ECT is generally underutilized and is often relegated to being a last-resort intervention in cases of medication non-response. However, the subjective report as well as the objective improvement per the PCL- 5 scale in this case suggests that ECT can aid in the treatment of TRN. Furthermore, the impact that ECT may have on the limbic symptom suggests the potential benefit in addressing TRN that are a result of a dysfunctional limbic symptom. However, further research is needed to determine the efficacy of ECT in TRN and in determining the specific mechanisms involved in reducing nightmares.

\section{Additional Information \\ Disclosures}

Human subjects: Consent was obtained or waived by all participants in this study. Conflicts of interest: In compliance with the ICMJE uniform disclosure form, all authors declare the following: Payment/services info: All authors have declared that no financial support was received from any organization for the submitted work. Financial relationships: All authors have declared that they have no financial relationships at present or within the previous three years with any organizations that might have an interest in the submitted work. Other relationships: All authors have declared that there are no other relationships or activities that could appear to have influenced the submitted work.

\section{References}

1. American Psychiatric Association: Diagnostic and Statistical Manual of Mental Disorders, 5th Edition . American Psychiatric Association, Washington, DC; 2013. 10.1176/appi.books.9780890425596

2. Rytwinski NK, Scur MD, Feeny NC, Youngstrom EA: The co-occurrence of major depressive disorder among individuals with posttraumatic stress disorder: a meta-analysis. J Trauma Stress. 2013, 26:299-309. $10.1002 /$ jts. 21814

3. Lavie P: Sleep disturbances in the wake of traumatic events . N Engl J Med. 2001, 345:1825-1832. 10.1056/NEJMra012893

4. Akkaoui MA, Lejoyeux M, d'Ortho MP, Geoffroy PA: Nightmares in patients with major depressive disorder, bipolar disorder, and psychotic disorders: a systematic review. J Clin Med. 2020, 9:3990. $10.3390 /$ jcm 9123990

5. Neylan TC, Marmar CR, Metzler TJ, et al.: Sleep disturbances in the Vietnam generation: findings from a nationally representative sample of male Vietnam veterans. Am J Psychiatry. 1998, 155:929-933. 10.1176/ajp.155.7.929

6. North CS, Nixon SJ, Shariat S, Mallonee S, McMillen JC, Spitznagel EL, Smith EM: Psychiatric disorders among survivors of the Oklahoma City bombing. JAMA. 1999, 282:755-762. 10.1001/jama.282.8.755

7. Krakow B, Germain A, Warner TD, et al.: The relationship of sleep quality and posttraumatic stress to potential sleep disorders in sexual assault survivors with nightmares, insomnia, and PTSD. J Trauma Stress. 2001, 14:647-665. 10.1023/A:1013029819358

8. Youssef NA, McCall WV, Andrade C: The role of ECT in posttraumatic stress disorder: a systematic review . Ann Clin Psychiatry. 2017, 29:62-70.

9. Nielsen TA, Stenstrom P: What are the memory sources of dreaming? . Nature. 2005, 437:1286-1289. 10.1038/nature04288

10. Forbes D, Creamer M, Bisson JI, et al.: A guide to guidelines for the treatment of PTSD and related conditions. J Trauma Stress. 2010, 23:537-552. 10.1002/jts.20565

11. Krakow B, Hollifield M, Johnston L, et al.: Imagery rehearsal therapy for chronic nightmares in sexual assault survivors with posttraumatic stress disorder: a randomized controlled trial. JAMA. 2001, 286:537545. 10.1001/jama.286.5.537

12. Miller KE, Davis JL, Balliet NE: Taking control: examining the influence of locus of control on the treatment of nightmares and sleep impairment in veterans. Mil Behav Health. 2014, 2:337-342. 10.1080/21635781.2014.963763

13. Raskind MA, Peskind ER, Hoff DJ, et al.: A parallel group placebo controlled study of prazosin for trauma nightmares and sleep disturbance in combat veterans with post-traumatic stress disorder. Biol Psychiatry. 2007, 61:928-934. 10.1016/j.biopsych.2006.06.032

14. Leiknes KA, Jarosh-von Schweder L, Høie B: Contemporary use and practice of electroconvulsive therapy worldwide. Brain Behav. 2012, 2:283-344. 10.1002/brb3.37

15. Pompili M, Lester D, Dominici G, et al.: Indications for electroconvulsive treatment in schizophrenia: a systematic review. Schizophr Res. 2013, 146:1-9. 10.1016/j.schres.2013.02.005

16. Tendolkar I, van Beek M, van Oostrom I, Mulder M, Janzing J, Voshaar RO, van Eijndhoven P: Electroconvulsive therapy increases hippocampal and amygdala volume in therapy refractory depression: a longitudinal pilot study. Psychiatry Res. 2013, 214:197-203. 10.1016/j.pscychresns.2013.09.004

17. Fosse R, Read J: Electroconvulsive treatment: hypotheses about mechanisms of action . Front Psychiatry. 


\section{Cureus}

2013, 4:94. 10.3389/fpsyt.2013.00094

18. Takano H, Motohashi N, Uema T, et al.: Changes in regional cerebral blood flow during acute electroconvulsive therapy in patients with depression: positron emission tomographic study. Br J Psychiatry. 2007, 190:63-68. 10.1192/bjp.bp.106.023036

19. Dukart J, Regen F, Kherif F, et al.: Electroconvulsive therapy-induced brain plasticity determines therapeutic outcome in mood disorders. Proc Natl Acad Sci U S A. 2014, 111:1156-1161. 10.1073/pnas.1321399111

20. Ahmadi N, Moss L, Simon E, Nemeroff CB, Atre-Vaidya N: Efficacy and long-term clinical outcome of comorbid posttraumatic stress disorder and major depressive disorder after electroconvulsive therapy. Depress Anxiety. 2016, 33:640-647. 10.1002/da.22451

21. Andrade C, McCall WV, Youssef NA: Electroconvulsive therapy for post-traumatic stress disorder: efficacy, mechanisms and a hypothesis for new directions. Expert Rev Neurother. 2016, 16:749-753.

10.1080/14737175.2016.1179114 